\title{
Dexamethasone Implant Improves Anatomic Response to Anti-VEGF Therapy in Treatment- Resistant Polypoidal Choroidal Vasculopathy
}

\section{Kushanth Mallikarjun}

Washington University in St. Louis

Raja Narayanan ( $\square$ narayanan@lvpei.org)

L V Prasad Eye Institute

\section{Rajiv Raman}

Sankara Nethralaya

\section{Ashik Mohamed}

L V Prasad Eye Institute

\section{Mahesh Shanmugam}

Sankara Hospitals, Bengaluru

\section{Rajendra Apte}

Washington University in St. Louis

\section{Srikant Padhy}

L V Prasad Eye Institute

\section{Research Article}

Keywords: Dexamethasone intravitreal implant, Anti-VEGF, Treatment resistance, Polypoidal choroidal vasculopathy

Posted Date: March 12th, 2021

DOI: https://doi.org/10.21203/rs.3.rs-282965/v1

License: (c) (1) This work is licensed under a Creative Commons Attribution 4.0 International License. Read Full License

Version of Record: A version of this preprint was published at International Ophthalmology on November 9th, 2021. See the published version at https://doi.org/10.1007/s10792-021-02113-4. 


\section{Dexamethasone implant improves anatomic response to anti-VEGF therapy in treatment-resistant Polypoidal Choroidal Vasculopathy}

Kushanth Mallikarjun, B.S. ${ }^{1}$, Raja Narayanan, M.D., M.B.A. ${ }^{2}$, Rajiv Raman,M.D. ${ }^{3}$, Ashik Mohamed,M.B.B.S. ${ }^{2}$, Mahesh P. Shanmugam, F.R.C.S. Ed, Ph.D. ${ }^{4}$, Rajendra S. Apte, M.D., Ph.D. ${ }^{1}$, Srikant Padhy, M.D. ${ }^{5}$

${ }^{1}$ Washington University School of Medicine in St. Louis, Missouri, USA; ${ }^{2}$ Smt. Kanuri

Santhamma Centre for Vitreoretinal Diseases, L.V. Prasad Eye Institute, India; ${ }^{3}$ Shri

Bhagwan Mahavir Vitreoretinal Services, Sankara Nethralaya, Chennai, India; ${ }^{4}$ Sankara Eye Hospitals, Bengaluru, India; ${ }^{5}$ L.V. Prasad Eye Institute, Bhubaneswar, India

Running Title: Dexamethasone Implant in PCV

This study was presented in part as free paper at the annual meeting of American Society of Retina Specialists, July 26, 2020

\section{Address for Correspondence:}

Raja Narayanan, M.D., M.B.A.

Suven Clinical Research Centre

L.V. Prasad Eye Institute

L.V. Prasad Marg, Banjara Hills

Hyderabad

Telangana 500034 
Email: narayanan@lvpei.org

Key words: Polypoidal choroidal vasculopathy; dexamethasone implant; visual acuity; optical coherence tomography; intravitreal injection 


\section{Abstract}

Background: A significant proportion of eyes with polypoidal choroidal vasculopathy (PCV) can be resistant to anti-vascular endothelial growth factor (VEGF) injections. We evaluated the efficacy of combination of Dexamethasone intravitreal implant (DXI) and anti-VEGF therapy in eyes resistant to anti-VEGF monotherapy.

Materials and Methods: In this retrospective study, polypoidal choroidal vasculopathy (PCV) resistant to anti-VEGF injections were additionally injected with a Dexamethasone implant along with an anti-VEGF agent. Best-corrected visual acuity (BCVA), slit lamp examination, intraocular pressure, fundus evaluation and optical coherence tomography (OCT) data were analyzed. Anatomical response on OCT was the primary outcome measure. Gain in visual acuity, and injection-free interval after the Dexamethasone implant were evaluated as secondary outcome measures.

Results: Twelve eyes of 11 patients were included in the study. The mean age of patients was $70.2 \pm 11.8$ years, and there were 8 females. The mean follow-up duration after DXI was $24.5 \pm 11$ months. The mean number of anti-VEGF injections before DXI was $4.2 \pm$ 1.9. The mean injection-free interval in these patients after DXI was $4.7 \pm 0.6$ months, which was significantly greater than the pre-injection mean of $1.6 \pm 0.4$ months $(p<0.001)$. The median log MAR BCVA immediately prior to DXI was 0.41 (Interquartile Range IQR 0.30-1.02) and after injection was 0.60 (IQR 0.27-1.03), which was not significant $(p=0.59)$. The median OCT thickness after DXI in was 305 microns (IQR 206-417), which was significantly less than the pre-injection OCT thickness of 547 microns (IQR 432-685) 
$(p=0.005)$. The mean IOP before DXI was $14.7 \pm 2.3 \mathrm{~mm} \mathrm{Hg}$, and after the injection was $14.3 \pm 2.7 \mathrm{~mm} \mathrm{Hg}(\mathrm{p}=0.36)$.

Conclusions: Dexamethasone implant combined with anti-VEGF treatment can prolong the treatment-free interval in eyes with PCV resistant to anti-VEGF injection, while maintaining visual acuity. 
Key Words: Dexamethasone intravitreal implant; Anti-VEGF; Treatment resistance; Polypoidal choroidal vasculopathy 


\section{Introduction}

Intravitreal injection of anti-vascular endothelial growth factor (VEGF) is the standard of care for choroidal neovascular membrane (CNVM) due to neovascular agerelated macular degeneration (nAMD) and polypoidal choroidal vasculopathy (PCV). ${ }^{[1-4]}$ However, a significant portion of eyes treated with anti-VEGF show little or no response to the therapy. ${ }^{[5,6]}$ Estimates of the prevalence of this anti-VEGF resistance among eyes with CNVM vary significantly in the literature based on anti-VEGF drug of choice, specific sub-type diagnosis such as nAMD or PCV, treatment style (prn vs treat-and-extend vs monthly regimen) and the ethnic population. ${ }^{[6-9]}$

Various inflammatory cytokines, such as macrophage derived chemokine (MDC), interleukins, and monocyte chemotactic protein levels were significantly higher in PCV patients have been shown to be elevated in eyes with PCV. ${ }^{[10]}$ Pharmacologic therapies which reduce inflammation in the eye may improve the outcomes in eyes with PCV. Dexamethasone implant (Ozurdex (DXI); Allergan, Irvine, California, USA), combined with anti-VEGF therapy, could improve the response to anti-VEGF agents in treatmentresistant PCV. A study by Kuppermann et al combining DXI with anti-VEGF agents in patients with nAMD demonstrated that the injection-free interval in patients receiving DXI along with anti-VEGF therapy was significantly longer than in patients receiving placebo with anti-VEGF therapy. ${ }^{[1]}$ However, the LuceDex trial did not show any difference in the number of injections between combination therapy and anti-VEGF monotherapy, although there was more visual gain in the combination therapy arm. ${ }^{[12]} \mathrm{A}$ few other studies have evaluated the efficacy of a combination treatment of DXI and anti-VEGF in nAMD patients resistant to anti-VEGF, but with contrasting outcomes. ${ }^{[13-15]}$ None of these 
patients included PCV. The purpose of this study was to evaluate visual outcomes and retreatment intervals after combination of Dexamethasone intravitreal implant (DXI) and anti-VEGF therapy in eyes resistant to anti-VEGF therapy in PCV.

\section{Methods}

The study protocol adhered to the tenets of the Declaration of Helsinki and was approved by the Institutional Review Board of L.V. Prasad Eye Institute. All study participants gave a written informed consent before enrollment. Patients were recruited from April 2017 through August 2019.

\section{Patient eligibility}

Inclusion criteria:

Key eligibility criteria included: (1) CNVM due to PCV; (2) minimum of 3 consecutive monthly anti-VEGF injections in the previous 12 weeks with $\leq 10 \%$ reduction in central sub-field thickness;

Exclusion criteria:

Key exclusion criteria included: (1) decrease in visual acuity due to causes other than PCV; (2) presence of glaucoma or steroid response; (3) use of intraocular or periocular corticosteroids in the study eye within the previous 6 months; (4) previous vitreoretinal surgery in the study eye or anticipated surgery within 12 months of enrollment.

Eyes were injected with 1) intravitreal dexamethasone implant (Ozurdex, Allergan, Irvine, CA, USA) and (2) $1.25 \mathrm{mg}$ bevacizumab, $0.5 \mathrm{mg}$ ranibizumab, or $1.25 \mathrm{mg}$ ziv-aflibercept. Patients were evaluated again every month. Patients were re-injected with intravitreal anti-VEGF if there was persistent intravitreal or subretinal fluid at any visit. 


\section{Ocular Evaluation}

All participants had a comprehensive ocular examination including best-corrected visual acuity (BCVA) testing, dilated fundus examination with slit-lamp biomicroscopy, color fundus photography, fundus fluorescein (FFA) and indocyanine green angiography (ICGA, Heidelberg Engineering GmbH, Heidelberg, Germany) and OCT (Triton DRI, Topcon, Japan). Patients underwent OCT at subsequent visits.

\section{Intravitreal injections}

Intravitreal injections were administered using a strict aseptic technique under topical anesthesia in an outpatient procedure room. Intravitreal injections were performed with 29-gauge needle inserted through the inferotemporal pars plana, $4 \mathrm{~mm}$ posterior to the limbus in phakic eyes and $3.5 \mathrm{~mm}$ in pseudophakic eyes. In eyes receiving combination treatment, DXI was injected after the anti-VEGF injection in the same sitting.

\section{Outcome measures}

Since this study was performed in resistant PCV, anatomical response on OCT was the primary outcome measure. Gain in visual acuity and injection-free interval after the dexamethasone implant were evaluated as secondary outcome measures.

\section{Statistical Analysis}

Data were analyzed using STATA version 16.1 (College Station, Texas, USA). The continuous data were checked for the normality of distribution by Shapiro-Wilk test and described in mean \pm standard deviation if normally distributed and median with interquartile range (IQR) if otherwise. Pre-and post-injection BCVA and OCT thickness were compared by Wilcoxon signed-rank test, while IOP was compared by Paired t-test. 
Categorical data were described in proportions and compared pre- and post-injection by McNemar test. Relationships between continuous data were assessed by Spearman correlation. Kaplan-Meier survival analysis was used to estimate the probability of first injection after DXI. A p-value of $<0.05$ was considered statistically significant.

\section{Results}

Twelve eyes of 11 patients were included in the study. The mean age of patients was $70.2 \pm 11.8$ years, and there were 8 females. The mean follow-up duration after DXI was $24.5 \pm 11$ months (Table 1). All eyes with PCV were confirmed by ICGA. The mean number of anti-VEGF injections before implant was $4.2 \pm 1.9$. All eyes received an injection along with DXI; bevacizumab in 2 eyes, ranibizumab in 4 eyes, and zivaflibercept in 6 eyes.

Visual acuity

The median BCVA before DXI injection was 0.41 (IQR,0.30-1.02), and 1 month after the injection was $0.40(\mathrm{IQR}, 0.30-1.05 ; \mathrm{p}=0.75)$. At the last visit, there was no significant change $(p=0.59)$ in BCVA with a median of 0.60 (IQR, 0.27-1.03). (Figure 1)

\section{$\underline{\text { Retinal thickness }}$}

The median OCT thickness before injection was 547 microns (IQR, 432-685), which decreased to 305 microns (IQR, 206-417) with a significant value $(p=0.005)$. The median change in OCT thickness was 207 microns (IQR, 30-559). (Figure 2) There was a significant $\left(p=0.004 ; r_{s}=0.74\right)$ positive correlation between pre-injection OCT thickness and change in OCT thickness in eyes with PCV.

\section{$\underline{\text { Retinal fluid compartments }}$}


The proportion of eyes with PCV having pigment epithelial detachment (PED) before injection was $76.9 \%$, which reduced to $53.8 \%$ and $30.8 \%$ after $1^{\text {st }}$ month and final followup respectively. At the last visit, the proportion of eyes with PED was $7.7 \%$ and a statistical significance $(p=0.03)$ was noted in the reduction of PED in eyes with PCV. The proportion of eyes having intra-retinal fluid and sub-retinal fluid was also reduced at the last visit which was significant $(p=0.01)$. (Figure 3$)$

Figures 4 and 5 show representative OCT images from two patients who initially showed no response to anti-VEGF monotherapy, yet showed good response and reduction in CRT after combination treatment with DXI and anti-VEGF. Ozurdex was not repeated in any patient, as this was a pilot study.

The mean injection-free interval in these patients after DXI was $4.7 \pm 0.6$ months, which was significantly greater than the pre-injection mean of $1.6 \pm 0.4$ months $(p<0.001)$. The injection-free interval was not correlated with number of injections $(p=0.23)$ and preinjection OCT thickness $(p=0.56)$. The survival analysis estimates of first injection after DXI were $20 \%$ at 3 months, $65 \%$ at 6 months, and $75.6 \%$ at 12 months. (Figure 6 )

\section{Discussion}

Intravitreal steroid injections in resistant nAMD have been studied with variable results. ${ }^{[13-19]}$ Inflammation is known to play a key role in the pathogenesis of PCV and nAMD. ${ }^{[10,20]}$ Oxidative stress has been shown to initiate the assembly of inflammasome complexes in the Retinal Pigment Epithelium. ${ }^{[21]}$ Complement components C3a and C5a have been shown to promote CNVM. ${ }^{[22,23]}$ Corticosteroids counteract macrophages and 
related cytokines involved in inflammation and neovascularization. ${ }^{[24,25]}$ There is also evidence that combination of intravitreal corticosteroids and anti-VEGF agents can decrease tachyphylaxis, or the decline in potency of these medications after a number of injections. ${ }^{[26]}$

Though intravitreal dexamethasone could have substantial impact, it is cleared from the eye quickly, with a half-life of less than 3.5 hours. ${ }^{[27]}$ In contrast, the Dexamethasone intravitreal implant used in this study has biological activity in the eye for 4-6 months. ${ }^{[28]}$ A small retrospective study on anti-VEGF resistant eyes also found that CRT declined significantly, which was held up until 3 months post-implantation. ${ }^{[13]}$ This study also reported a total absence of any fluid 3 months after dexamethasone injection in $71.4 \%$ of their patients.

The stability of BCVA after DXI in these anti-VEGF resistant PCV patients in our study is understandable, given the fact that DXI was given after anti-VEGF therapy had been started. It is possible that the usual decline in BCVA for these patients associated with the normal course of PCV on anti-VEGF therapy has been slowed by DXI. If this is the case, vision stabilization in these patients with a reduced burden of injections would indeed be a desirable outcome.

Given that the frequency of anti-VEGF injections poses a huge burden on patients, reducing the burden of injections by increasing the time between injections, must be a goal of future therapy. ${ }^{[29]}$ However, for the significant portion of patients who fail to respond to anti-VEGF therapy, combination with steroids could potentially have a role in reducing the resistance to anti-VEGF therapy. Our study in eyes with PCV demonstrates that the burden of frequent injections can be reduced with the addition of a steroid 
extended-release implant by significantly increasing the injection-free interval to more than 4 months. This is a substantially longer duration compared to other studies in nAMD, and promises to reduce the treatment burden significantly. Our study shows that intravitreal steroid implants can also lead to significant reduction of macular edema. In all but two patients in this study, Central Retinal Thickness (CRT) declined during the month after the implant was placed. Significantly fewer patients had any fluid in any compartment on OCT after the implant.

The limitations of our study include the relatively small sample size, as well as the fact that it was a retrospective study. A larger, randomized, prospective study of PCV would be more clearly able to evaluate the effects of the DXI implant used in combination with anti-VEGF therapy, as well as determine what biomarkers or trends are unique to patients who still fail to respond significantly to this dual therapy. Our small sample could not evaluate the impact of high reflective dots in the choroid or the retina. Strengths of our study include significant increase of the injection-free interval after steroid implant in resistant cases. There were no significant safety concerns in this small study,

\section{Conclusion}

This study shows that the addition of intravitreal dexamethasone implant in PCV, resistant to anti-VEGF, could stabilize visual acuity and partially restore foveal anatomy. Further studies with larger sample sizes, should also look into biomarkers of eyes resistant to anti-VEGF,

\section{List of Abbreviations}

BCVA Best Corrected Visual Acuity




\begin{tabular}{ll}
\hline CNVM & Choroidal Neovascular Membrane \\
\hline CRT & Central Retinal Thickness \\
\hline DXI & Dexamethasone Implant \\
\hline FFA & Fundus Fluorescein Angiography \\
\hline ICGA & Indocyanine Green Angiography \\
\hline IOP & Intraocular Pressure \\
\hline IQR & Interquartile Range \\
\hline ME & Macular Edema \\
\hline nAMD & Neovascular Age Related Macular Degeneration \\
\hline PCV & Polypoidal Choroidal Vasculopathy \\
\hline PED & Pigment Epithelial Detachment \\
\hline OCT & Optical Coherence Tomography \\
\hline VEGF & Vascular Endothelial Growth Factor \\
\hline
\end{tabular}

\section{Declarations}

\section{Ethics approval and consent to participate}

A written consent was obtained from patients in accordance with the declaration of Helsinki. The study was approved by respective Ethics Committee of L.V. Prasad Eye Institute.

\section{Consent for publication}

Not Applicable 


\section{Availability of data and materials}

The datasets generated during and analyzed during the current study are are available from the corresponding author on reasonable request.

\section{Competing interests}

None of the authors have any competing interests.

\section{Funding}

Hyderabad Eye Research Foundation and India Alliance DBT Wellcome Trust. 


\section{Authors' contribution}

$\mathrm{RN}, \mathrm{KM}$ contributed to data collection, analysis and interpretation data, preparation of the manuscript and provided major revisions to the manuscript. RR, AM, MPS, RA and SP contributed to interpretation and final analysis of manuscript. AM and KM performed statistical analysis. All authors participated in the development and writing of the manuscript and approved the final article for publication.

\section{Acknowledgements}

Hyderabad Eye Research Foundation and India Alliance DBT Wellcome Trust. 


\section{References}

1. Koh A, Lee WK, Chen LJ, Chen SJ, Hashad Y, Kim H, et al. EVEREST study: efficacy and safety of verteporfin photodynamic therapy in combination with ranibizumab or alone versus ranibizumab monotherapy in patients with symptomatic macular polypoidal choroidal vasculopathy. Retina. 2012;32(8):1453-64.

2. Lee WK, lida T, Ogura Y, Chen SJ, Wong TY, Mitchell P, et al. Efficacy and Safety of Intravitreal Aflibercept for Polypoidal Choroidal Vasculopathy in the PLANET Study: A Randomized Clinical Trial. JAMA Ophthalmol. 2018;136(7):786-93.

3. Martin DF, Maguire MG, Fine SL, Ying GS, Jaffe GJ, Grunwald JE et al. Ranibizumab and bevacizumab for treatment of neovascular age-related macular degeneration: two-year results. Ophthalmology. 2012;119:1388-98.

4. Heier JS, Brown DM, Chong V, Korobelnik JF, Kaiser PK, Nguyen QD et al. Intravitreal aflibercept (VEGF trap-eye) in wet age-related macular degeneration. Ophthalmology. 2012;119:2537-48.

5. Lux A, Llacer $H$, Heussen FM, Joussen AM. Non-responders to bevacizumab (Avastin) therapy of choroidal neovascular lesions. Br J Ophthalmol. 2007;91:131822.

6. Krebs I, Glittenberg C, Ansari-Shahrezaei S, Hagen S, Steiner I, Binder S. Nonresponders to treatment with antagonists of vascular endothelial growth factor in agerelated macular degeneration. Br J Ophthalmol. 2013;97:1443-6. 
7. Suzuki M, Nagai N, Izumi-Nagai K, Shinoda H, Koto T, Uchida A et al. Predictive factors for non-response to intravitreal ranibizumab treatment in age-related macular degeneration. Br J Ophthalmol. 2014;98:1186-91.

8. Kokame GT, deCarlo TE, Kaneko KN, Omizo JN, Lian R. Anti-Vascular Endothelial Growth Factor Resistance in Exudative Macular Degeneration and Polypoidal Choroidal Vasculopathy. Ophthalmology Retina. 2019;3:744-52.

9. Yang S, Zhao J, Sun X. Resistance to anti-VEGF therapy in neovascular age-related macular degeneration: a comprehensive review. Drug design, development and therapy. 2016;10:1857.

10. Agrawal R, Balne PK, Wei X, Bijin VA, Lee B, Ghosh A, et al. Cytokine Profiling in Patients With Exudative Age-Related Macular Degeneration and Polypoidal Choroidal Vasculopathy. Invest Ophthalmol Vis Sci. 2019;60(1):376-82.

11. Kuppermann BD, Goldstein M, Maturi RK, Pollack A, Singer M, Tufail A et al. Dexamethasone intravitreal implant as adjunctive therapy to ranibizumab in neovascular age-related macular degeneration: a multicenter randomized controlled trial. Ophthalmologica. 2015;234:40-54.

12. Ranchod TM, Ray SK, Daniels SA, Leong CJ, Ting TD, Verne AZ. LuceDex: a prospective study comparing ranibizumab plus dexamethasone combination therapy versus ranibizumab monotherapy for neovascular age-related macular degeneration. Retina. 2013;33(8):1600-1604. 
13. Calvo P, Ferreras A, Al Adel F, Wang Y, Brent MH. Dexamethasone intravitreal implant as adjunct therapy for patients with wet age-related macular degeneration with incomplete response to ranibizumab. Br J Ophthalmol. 2015;99:723-6.

14. Rezar-Dreindl S, Eibenberger K, Buehl W, Georgopoulos M, Weigert G, Krall C et al. Role of additional dexamethasone for the management of persistent or recurrent neovascular age-related macular degeneration under ranibizumab treatment. Retina. 2017;37:962-70.

15. Todorich B, Thanos A, Yonekawa Y, Mane G, Hasbrook M, Thomas BJ et al. Simultaneous dexamethasone intravitreal implant and anti-vegf therapy for neovascular age-related macular degeneration resistant to anti-vegf monotherapy. Journal of vitreoretinal diseases. 2017;1:65-74.

16. Giancipoli E, Pinna A, Boscia F, Zasa G, Sotgiu G, Dore S et al. Intravitreal dexamethasone in patients with wet age-related macular degeneration resistant to anti-vegf: A prospective pilot study. J Ophthalmol. 2018.

17. Jonas JB, Libondi T, Golubkina L, Spandau UH, Schlichtenbrede F, Rensch F. Combined intravitreal bevacizumab and triamcinolone in exudative age-related macular degeneration. Acta Ophthalmol. 2010;88:630-4.

18. Tozer K, Roller AB, Chong LP, Sadda S, Folk JC, Mahajan VB et al. Combination therapy for neovascular age-related macular degeneration refractory to anti-vascular endothelial growth factor agents. Ophthalmology. 2013;120:2029-34.

19. Veritti D, Sarao V, Lanzetta P. Bevacizumab and triamcinolone acetonide for choroidal neovascularization due to age-related macular degeneration unresponsive to antivascular endothelial growth factors. J Ocul Pharmacol Ther. 2013;29:437-41. 
20. Kauppinen A, Paterno JJ, Blasiak J, Salminen A, Kaarniranta K. Inflammation and its role in age-related macular degeneration. Cell Mol Life Sci. 2016;73:1765-86.

21. Kauppinen A, Niskanen H, Suuronen T, Kinnunen K, Salminen A, Kaarniranta K. Oxidative stress activates NLRP3 inflammasomes in ARPE-19 cells-implications for age-related macular degeneration (AMD). Immunol Lett. 2012;147:29-33.

22. Nozaki M, Raisler BJ, Sakurai E, Sarma JV, Barnum SR, Lambris JD et al. Drusen complement components C3a and C5a promote choroidal neovascularization. Proc Natl Acad Sci U S A. 2006;103:2328-33.

23. Machalińska A, Dziedziejko V, Mozolewska-Piotrowska K, Karczewicz D, Wiszniewska B, Machaliński B. Elevated plasma levels of C3a complement compound in the exudative form of age-related macular degeneration. Ophthalmic Res. 2009;42:54-9.

24. Oliver A, Ciulla TA. Corticosteroids as antiangiogenic agents. Ophthalmol Clin North Am. 2006;19:345-51.

25. Oh H, Takagi H, Takagi C, Suzuma K, Otani A, Ishida K et al. The potential angiogenic role of macrophages in the formation of choroidal neovascular membranes. Invest Ophthalmol Vis Sci. 1999;40:1891-8.

26. Schaal S, Kaplan HJ, Tezel TH. Is there tachyphylaxis to intravitreal anti-vascular endothelial growth factor pharmacotherapy in age-related macular degeneration?. Ophthalmology. 2008;115:2199-205.

27. Kwak HW, D'Amico DJ. Evaluation of the retinal toxicity and pharmacokinetics of dexamethasone after intravitreal injection. Arch Ophthalmol. 1992;110:259-66. 
28. Chang-Lin JE, Attar M, Acheampong AA, Robinson MR, Whitcup SM, Kuppermann BD et al. Pharmacokinetics and pharmacodynamics of a sustained-release dexamethasone intravitreal implant. Invest Ophthalmol Vis Sci. 2011;52:80-6.

29. van Wijngaarden P, Coster DJ, Williams KA. Inhibitors of ocular neovascularization: promises and potential problems. JAMA. 2005;293:1509-13. 


\section{Figure Legends}

\section{Figure 1}

1A. Box plot comparison of Best-Corrected Visual Acuity (BCVA) expressed as a decimal before DXI (left) and at last follow up after DXI implantation (right). BCVA was maintained during this interval. 1B. Scatter plot showing that the final BCVA was correlated with baseline BCVA.

\section{Figure 2}

Box plot comparing Central Retinal Thickness (CRT) before DXI (left) and after DXI (right). There was a significant reduction in central retinal thickness before and after DXI implant.

Figure 3: Presence of retinal fluid in various compartments at baseline and after injection. There was a significant reduction in fluid in all compartments.

IRF: intraretinal fluid

SRF: subretinal fluid

PED: pigment epithelial detachment

\section{Figure 4}


The patient had received two monthly ranibizumab injections with no response ( $3 a$ and $3 b$ show OCT images after each injection). Figure $3 c$ and $3 d$ are OCT images taken after the combination of ranibizumab and DXI injections, which showed good response with no intra-retinal or sub-retinal fluid three weeks and two months after the injection, respectively.

\section{Figure 5}

The patient had received four bevacizumab and one ranibizumab injections with poor response. (Figure $4 \mathrm{a}$ shows OCT images after these injections). Figure $4 \mathrm{~b}$ and $4 \mathrm{c}$ show complete resolution of macular fluid after ranibizumab and DXI implant, one and six months later, respectively.

\section{Figure 6}

Kaplan Meier survival analysis estimate of risk of repeat anti-VEGF injection after DXI implant. 
Table 1: Demographic data of patients with PCV

\section{Characteristics}

Mean age (years)

Male

Female

Right eye (OD)

Left eye (OS)

Mean prior Anti-VEGFs ( $\mathrm{n}$ ) n (\%)

70.2

$4(33.3 \%)$

$8(66.6 \%)$

$5(41.6 \%)$

$7(58.3 \%)$

$4.2 \pm 1.9$ 
Figures
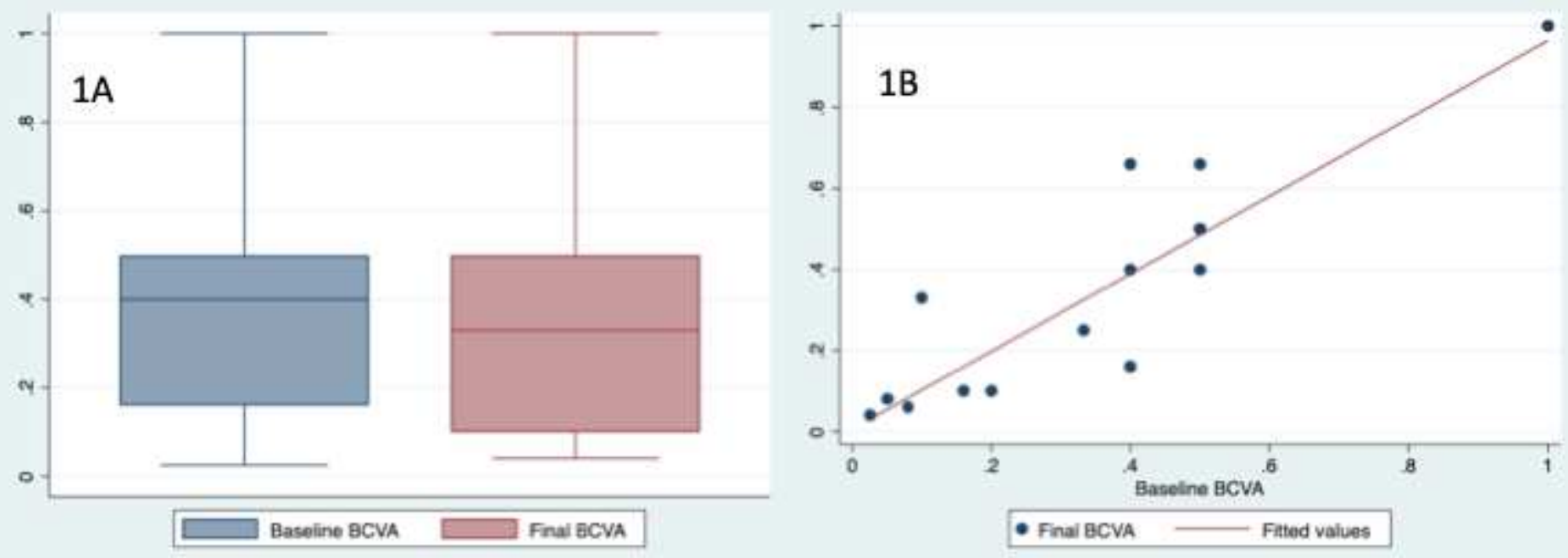

\section{Figure 1}

1A. Box plot comparison of Best-Corrected Visual Acuity (BCVA) expressed as a decimal before DXI (left) and at last follow up after DXI implantation (right). BCVA was maintained during this interval. 1B. Scatter plot showing that the final BCVA was correlated with baseline BCVA. 


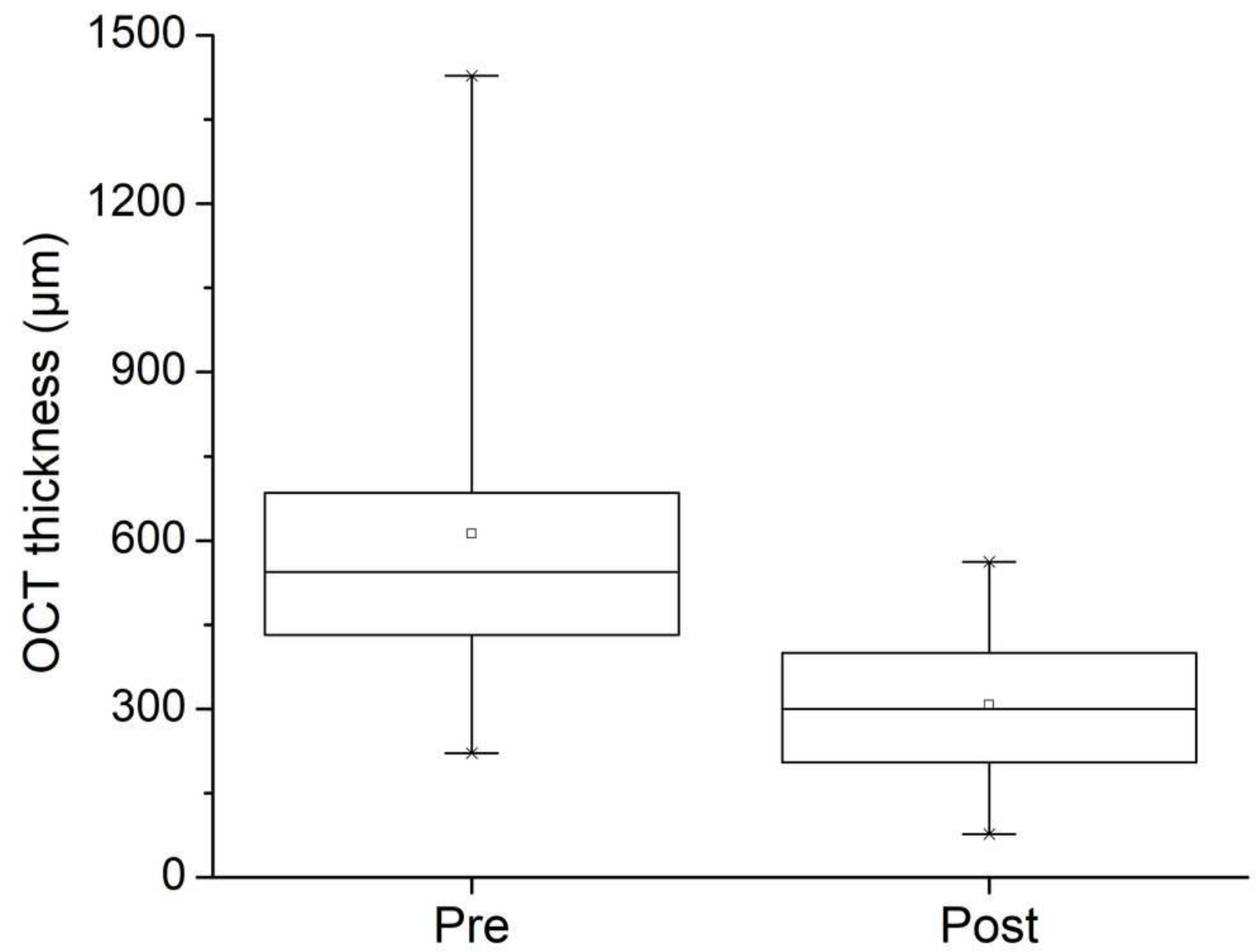

Figure 2

Box plot comparing Central Retinal Thickness (CRT) before DXI (left) and after DXI (right). There was a significant reduction in central retinal thickness before and after DXI implant. 


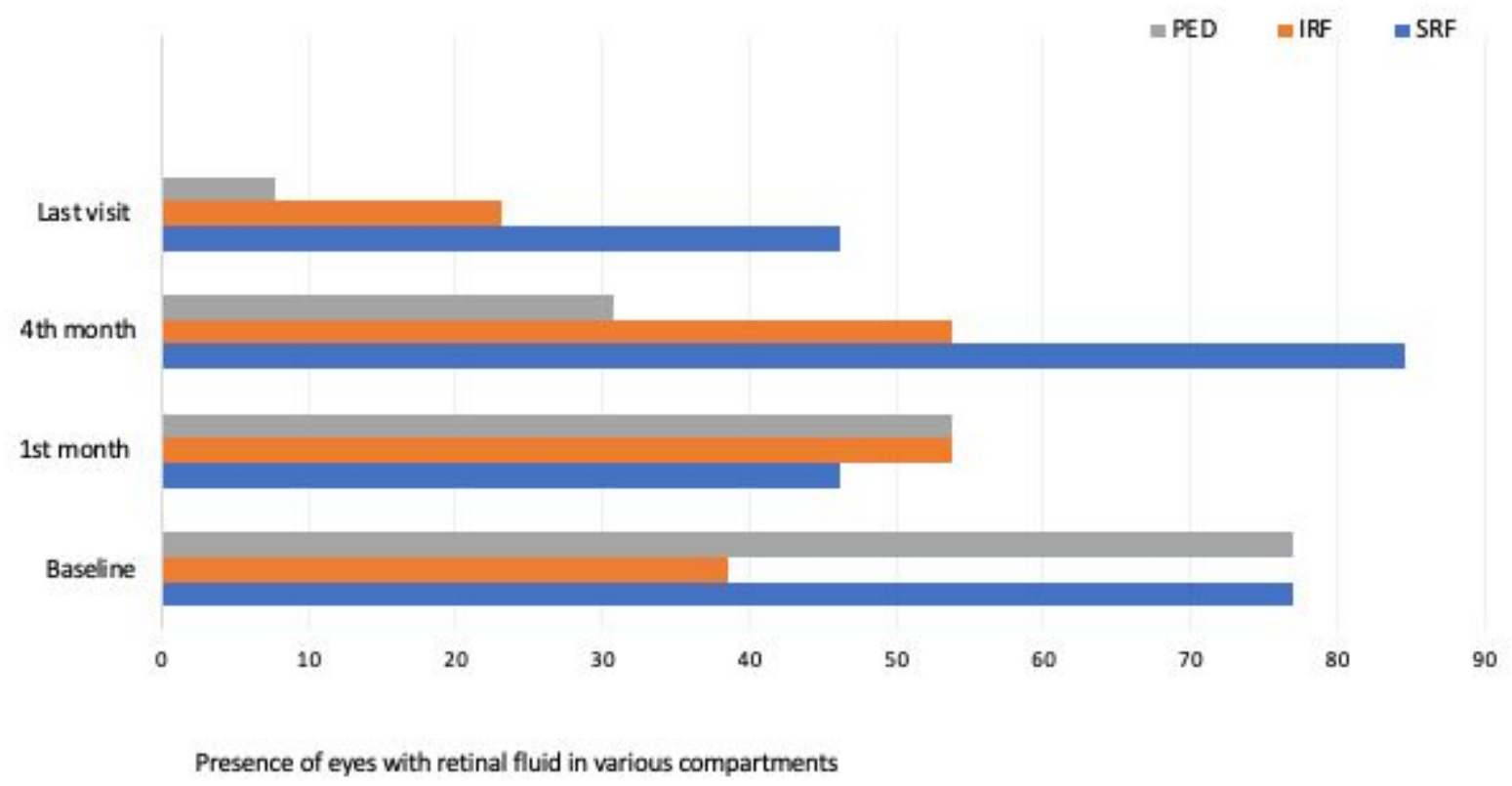

Figure 3

Presence of retinal fluid in various compartments at baseline and after injection. There was a significant reduction in fluid in all compartments. IRF: intraretinal fluid SRF: subretinal fluid PED: pigment epithelial detachment 


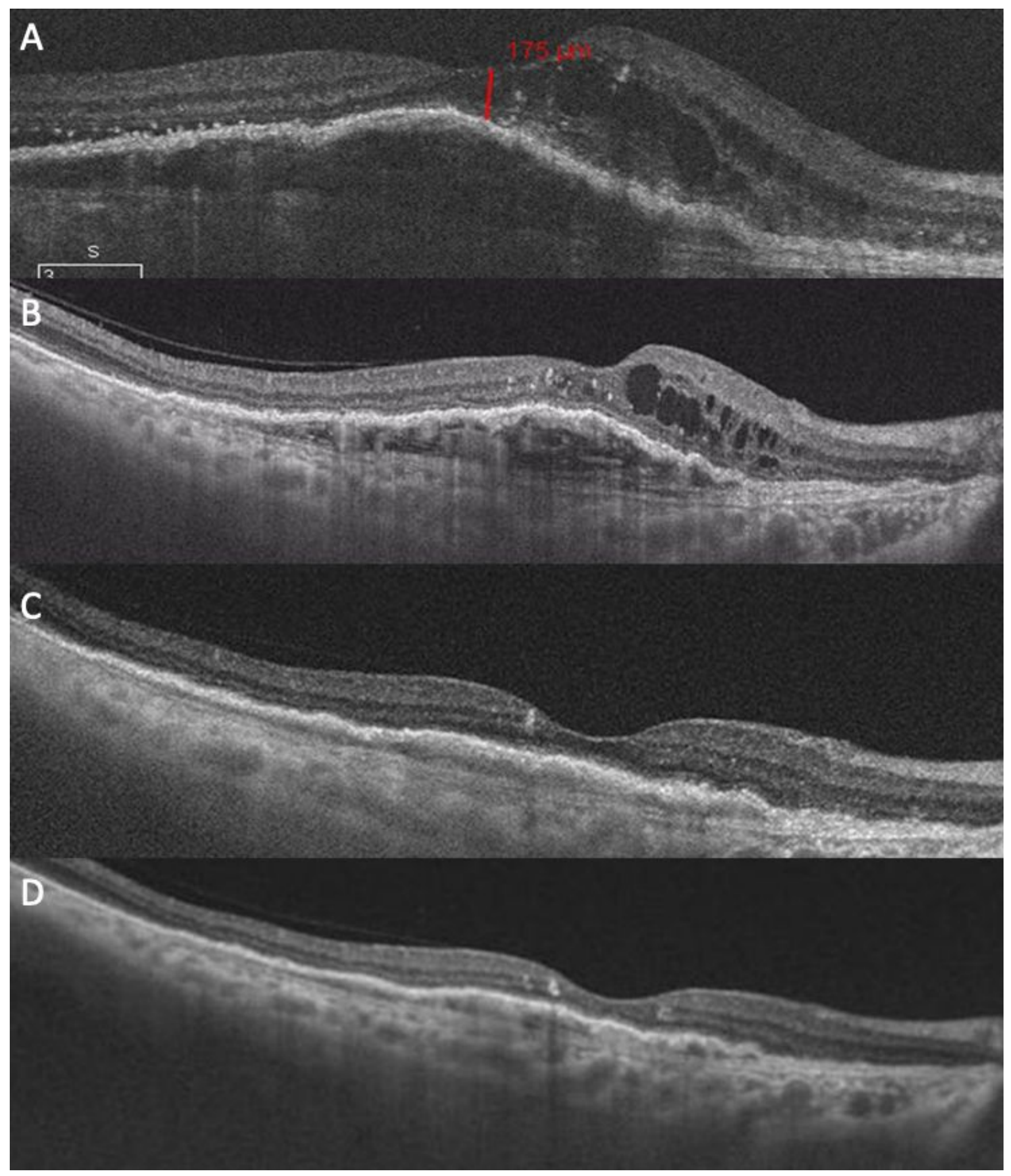

\section{Figure 4}

The patient had received two monthly ranibizumab injections with no response ( $3 \mathrm{a}$ and $3 \mathrm{~b}$ show OCT images after each injection). Figure $3 c$ and $3 d$ are OCT images taken after the combination of ranibizumab and DXI injections, which showed good response with no intra-retinal or sub-retinal fluid three weeks and two months after the injection, respectively. 


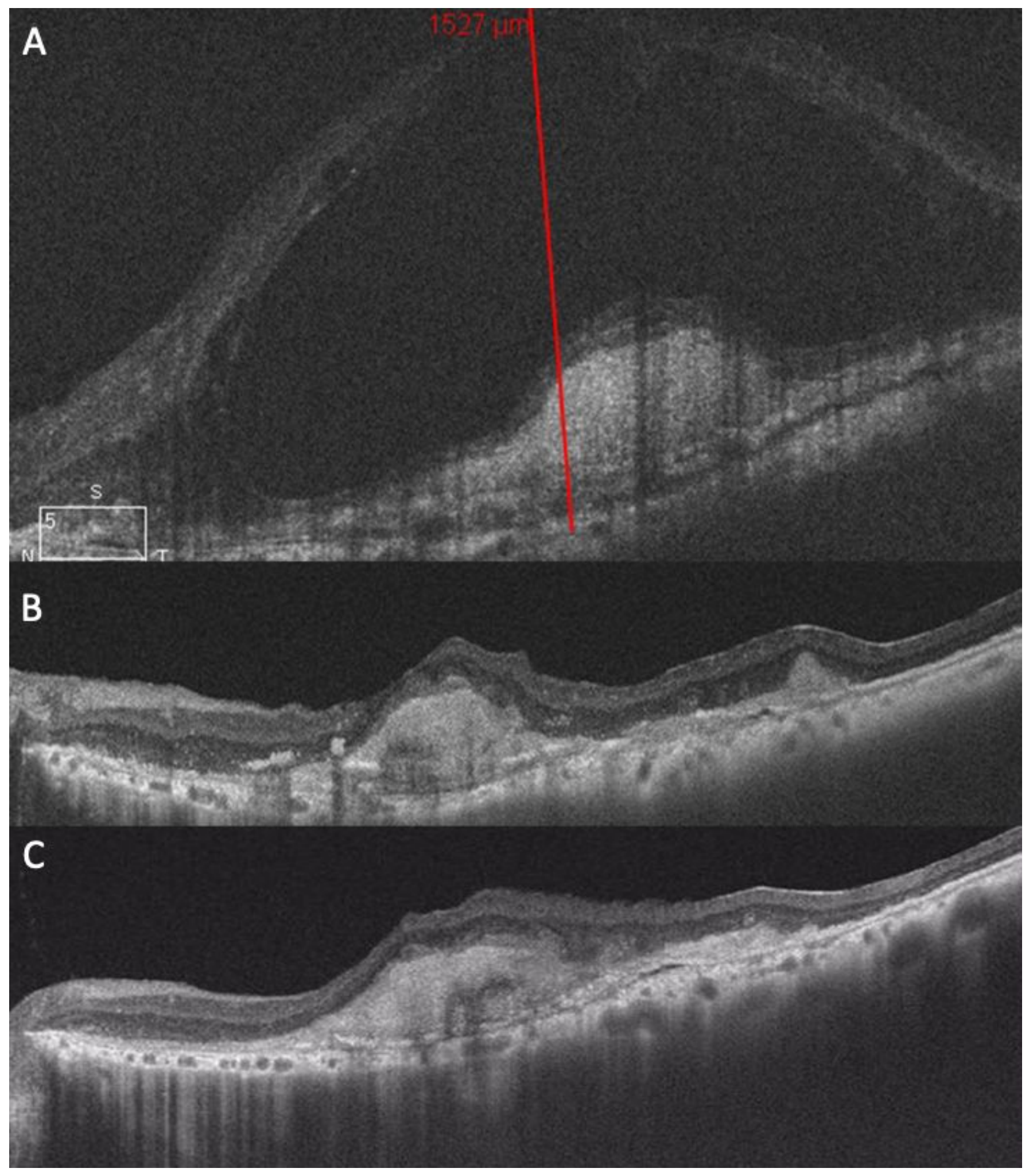

\section{Figure 5}

The patient had received four bevacizumab and one ranibizumab injections with poor response. (Figure 4a shows OCT images after these injections). Figure $4 \mathrm{~b}$ and $4 \mathrm{c}$ show complete resolution of macular fluid after ranibizumab and DXI implant, one and six months later, respectively. 


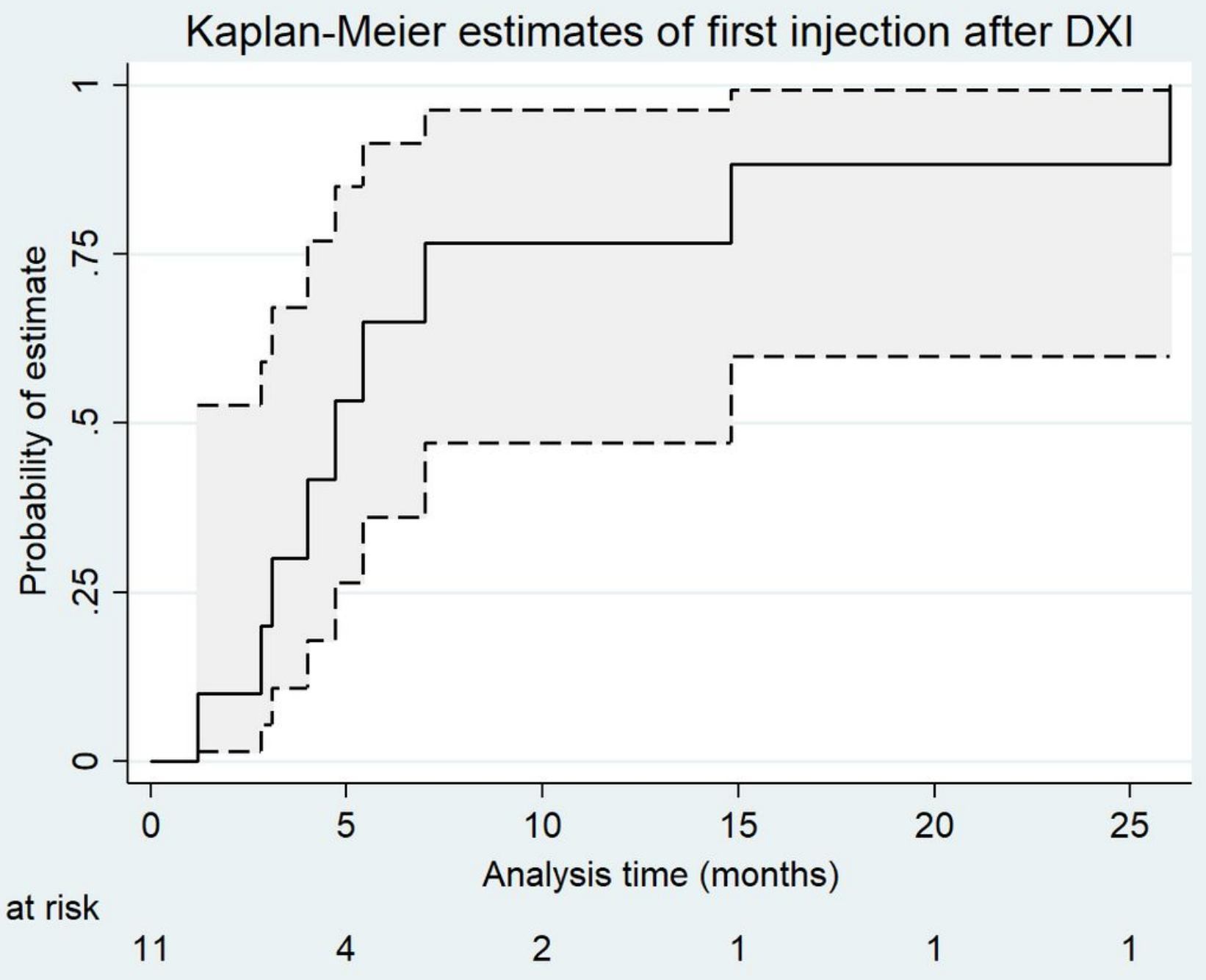

Figure 6

Kaplan Meier survival analysis estimate of risk of repeat anti-VEGF injection after DXI implant. 